Ann. Biol. anim. Bioch. Biophys., 1979, 19 (1B), 235-240.

\title{
Influence du niveau des apports énergétiques distribués à la brebis pendant la gestation sur le poids à la naissance ef la croissance des agneaux
}

par M. TISSIER, M. THERIEZ

Laboratoire de la Production ovine, I.N.R.A.

Theix, St Genès Champanelle, 63110 Beaumont.

Summary. Effects of ewe energy levels during pregnancy on lamb birth weight and growth rate.

The effect of ewe energy levels during pregnancy on lamb birth weight and growth rate were studied in two experiments of the same design carried out either at spring lambing in March or at autumn lambing in November. During mid-pregnancy (weeks 6 to 14), two types of body conditions (fat and lean) were achieved and combined with two energy levels (low and high : approximately 1.0 and 1.4 maintenance level) in late pregnancy (weeks 15 to 21) (fig. 1). The ewes bore single or twin fœtuses. During lactation they were fed at the same level according to maximum milk yield, depending on whether they were suckling a single or twin lambs. The main results are given in table 1.

1. There was no treatment effect on birth weight or growth rate of single lambs.

2. At spring lambing, the average birth weight of the twin lambs was significantly affected by the treatments at mid-pregnancy and overall in late pregnancy $(3,29 \mathrm{VS} 3,56 \mathrm{~kg}$ for the lean and fat group - P $<0,100 ; 3,01 \mathrm{VS} 3,84 \mathrm{~kg}$ for the low and high level $-\mathrm{P}<0,001)$. Also, the growth rote of these lambs, after weaning at 6 weeks, was significantly reduced when their mothers were restricted to the maintenance level in late pregnancy ( 260 VS $288 \mathrm{~g} /$ day for the low and high level $-P<0,025$ ).

3. These effects were not observed in ewes of the autumn lambing, but lamb birth weights were usually a liftle lower than in spring.

These results are discussed, with particular attention to seasonal effects which require more research, as these two experiments were not designed to test such effects.

\section{Introduction.}

De nombreux travaux ont été effectués pour déterminer les effets du niveau des apports énergétiques distribués pendant la gestation sur les performances des brebis, le poids à la naissance et la croissance de leurs agneaux. A la fin de la gestation, avec des niveaux d'apports extrêmes allant de 2,5 à 0,5 fois l'apport nécessaire pour l'entretien, Wallace (1948) avait obtenu des poids à la naissance d'agneaux nés doubles de 4,9 à 2,6 kg avec une diminution de leur vitalité et de leur capacité de croissance ; la production laitière des brebis était diminuée directement par la restriction énergétique, mais aussi indirectement par le manque de vitalité des agneaux. Plus récemment, 
différents auteurs, dont Peart (1967), Treacher (1970), Louca, Mavrogenis et Law or (1974) ont trouvé des différences dans le même sens, avec des écarts moins prononcés entre les niveaux d'apports énergétiques distribués. Toutes les données disponibles sont cependant peu concordantes quant aux récommandations énergétiques minimales à la fin de la gestation, et elles ont été obtenues pour la plupart sur des brebis agnelant une seule fois par an. Les résultats présentés dans cette note décrivent les effets sur le poids à la naissance ef la croissance des agneaux, de différents niveaux d'apports énergétiques au milieu et à la fin de la gestation distribués à des brebis conduites dans un système de 3 agnelages en 2 ans.

\section{Matériels et méthodes.}

Les effets des 2 niveaux d'apports à la mi-gestation ( $2^{\mathrm{e}}$ et $3^{\mathrm{e}}$ mois) combinés à 2 niveaux d'apports pendant la fin de la gestation ( 6 dernières semaines) ont été étudiés au cours de 2 expériences, l'une à l'agnelage de printemps, l'autre à l'agnelage d'automne. Chacune des expériences incluait 48 brebis adultes de race Limousine portant 1 ou 2 agneaux, logées et nourries en cases individuelles pendant les 7 dernières semaines de la gestation et les 6 semaines de la lactation, après lesquelles les agneaux sont sevrés. A la mi-gestation les niveaux d'apports alimentaires étaient constitués par du foin à volonté $+400 \mathrm{~g}$ d'aliment concentré (expérience 1) ou une charge au pâturage de 5 brebis à l'hectare (expérience 2) (lot gras : $G$ ) et du foin en quantité limitée $(1 \mathrm{~kg} / \mathrm{j})$ ou une charge au pâturage de 10 brebis à l'hectare (lot maigre : M) (fig. 1).

A la fin de la gestation, les niveaux d'apports énergétiques haut $(H)$ et bas $(B)$ apportaient respectivement 1,4 et 1,0 fois l'apport nécessaire pour l'entretien; les rations comprenaient du fourrage - foin (expérience 1) ou luzerne déshydratée (expérience 2) - et un aliment concentré (maïs et tourteau de soja), distribués en quantités différentes mais dans le même rapport quels que soient les niveaux $\mathrm{H}$ et $B$ (fig. 1). Les apports azotés ef minéraux étaient ajustés pour toutes les brebis selon les recommandations admises jusque-là.

L'interprétation statistique selon des plans factoriels nous a permis d'isoler et de tester, pour chacune des mesures effectuées (poids, productions laitières, etc...), les effets principaux et les interactions des facteurs : niveaux des apports et type de portée.

\section{Résultats et discussion.}

Effets du niveau des apports alimentaires distribués à la mi-gestation.

Aucun effet significatif $n$ 'a pu être mis en évidence dans le cas des portées simples.

Par contre, pour les portées doubles à l'agnelage de printemps, le poids des agneaux à la naissance du lot $M$ est d'environ 10 p. 100 inférieur à celui du lot $G$ $(P<0,10)$, bien que les deux niveaux d'apports n'aient pas entraîné de différences importantes $(2 \mathrm{~kg}$ environ, $\mathrm{P}<0,20)$ sur le poids des brebis (tabl. 1). La réduction observée du poids à la naissance n'a pas eu de conséquences significatives sur la croissance ultérieure des agneaux. Ces résultats sont en accord avec ceux de Everitt (1964, 


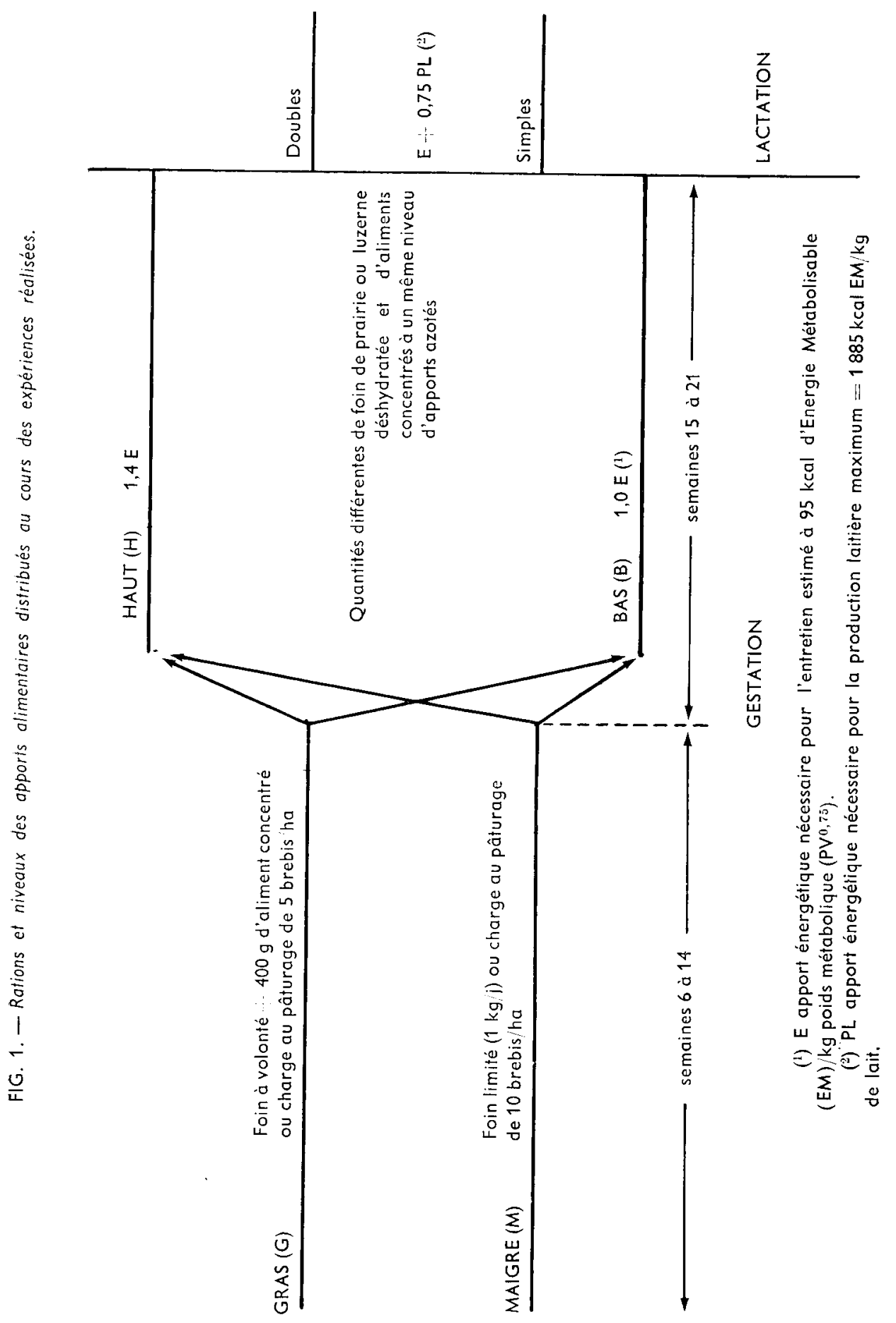


1967) et de Curll, Davidson et Freer (1975) qui ont observé des effets très marqués, particulièrement lorsque le niveau d'apports est insuffisant pour maintenir le poids des brebis. Dans ce cas, Everitt (1967) a observé un développement moindre des cotylédons ef du placenta.

Nous n'avons observé aucune différence significative à l'agnelage d'automne.

TABLEAU 1

Effets du niveau des apports énergétiques pendanf la gestation sur le poids à la naissance ef la croissance des agneaux après sevrage. Influence de la saison d'agnelage (brebis d̀ 2 agneaux)

\begin{tabular}{|c|c|c|c|c|c|}
\hline \multirow[b]{2}{*}{ Période } & \multirow[b]{2}{*}{$\begin{array}{l}\text { Lot ou } \\
\text { niveau } \\
\text { d'apport }\end{array}$} & \multicolumn{2}{|c|}{ Printemps } & \multicolumn{2}{|c|}{ Automne } \\
\hline & & $\begin{array}{l}\text { Poids des agneaux } \\
\text { à la naissance }\end{array}$ & $\begin{array}{c}\text { Croissance } \\
\text { après sevrage }\end{array}$ & $\begin{array}{l}\text { Poids des agneaux } \\
\text { à la naissance }\end{array}$ & $\begin{array}{c}\text { Croissance } \\
\text { après sevrage }\end{array}$ \\
\hline $\begin{array}{c}\text { mi- } \\
\text { gestation }\end{array}$ & $\begin{array}{c}\text { gras } \\
\text { maigre }\end{array}$ & $\begin{array}{ll}3,56 & \\
3,29 & P<0,1\end{array}$ & $\begin{array}{ll}277 & \\
271 & \text { NS }\end{array}$ & $\begin{array}{l}3,35 \\
3,22\end{array}$ NS & $\begin{array}{ll}228 & \\
232 & N S\end{array}$ \\
\hline $\begin{array}{l}\text { fin de } \\
\text { gestation }\end{array}$ & $\begin{array}{l}\text { haut } \\
\text { bas }\end{array}$ & $\begin{array}{l}3,84 \\
3,01\end{array}$ & $280 \quad \mathrm{P}<0,025$ & $\begin{array}{l}3,32 \\
3,27\end{array}$ NS & 240 NS \\
\hline
\end{tabular}

Effets du niveau des apports alimentaires distribués à la fin de la gestation.

A l'agnelage de printemps, le poids à la naissance des agneaux doubles est diminué de plus de 20 p. $100(3,01$ contre $3,84 \mathrm{~kg}, \mathrm{P}<0,001)$ alors que celui des simples n'est pas affecté significativement lorsque le niveau des apports passe de 1,4 E à 1,0 E. De plus, leur croissance après le sevrage (de 6 à 10 semaines) est plus faible de 10 p. 100 $(P<0,025$ (tabl. 1). Cette diminution du poids des agneaux doubles à la naissance est comparable à celles trouvées par Peart (1967), Treacher (1970) et Louca, Mavrogenis ef Lawlor (1974). La réduction observée de la croissance après le sevrage alors que les agneaux sont nourris à volonté, montre que leur potentiel de croissance peut être affecté définitivement par la sous-alimentation de la mère pendant la fin de la gestation ; l'hypothèse d'un tel effet avait été formulée par Everitt (1967) et Peart (1970). Par ailleurs, Wallace (1948) avait observé que, plus que tous les autres organes, le foie, la rate et le thymus des fœtus ont un poids très réduit dans le cas de sous-alimentation prononcée de la mère : il est possible que la réduction de poids de ces organes soit responsable de la diminution de croissance des agneaux.

Comme pour l'étude depuis la mi-gestation, les résultats trouvés à l'agnelage d'automne ne montrent pas de tels effets.

Pendant la période d'allaitement, même pour les portées doubles, on n'a pas observé d'effets résiduels des niveaux d'apports distribués pendant la fin de la gestation sur la production laitière des brebis et par conséquent sur la croissance de leurs agneaux pendant cette période. La variabilité associée à ces deux mesures, dont une 
partie est sans doute d'ordre génétique, est trop élevée (coefficient de variation résiduelle de 20 à 25 p. 100) pour que l'on puisse mettre en évidence des différences faibles.

\section{Influence de la saison d'agnelage, apports azotés.}

Les différences de poids de naissance et de croissance des agneaux consécutives aux écarts entre niveaux d'apports énergétiques ne peuvent pas être généralisés, car ils n'apparaissent pas toujours (notamment à l'agnelage d'automne) comme dans d'autres travaux disponibles dans la bibliographie. Une des raisons probables est que les brebis utilisent différemment leurs réserves corporelles ; en effet, avec des niveaux alimentaires comparables pendant la lactation, nous avons toujours observé que les pertes de poids des brebis pendant les 6 premières semaines étaient plus importantes à l'agnelage d'automne qu'à l'agnelage de printemps et nous avons confirmé récemment (Purroy, 1978) que les pertes de lipides étaient également plus marquées.

D'autre part, même si le niveau des apports énergétiques a une influence prépondérante particulièrement à la fin de la gestation, les apports azotés sont à considérer en eux-mêmes, et dans leurs interactions avec les apports énergétiques ; on sait que l'énergie comme l'azote des rations peuvent limiter les synthèses de protéines microbiennes dans le rumen (Jarrige, Journet et Vérité, 1978) et par conséquent les quantités de Protéines Digestibles dans l'Intestin (PDI) utilisées pour les dépenses d'entretien et de production, notamment pour la croissance des fœtus. D'ailleurs, à l'agnelage de printemps on a observé (Grizard et al., 1979) une réduction des teneurs sanguines de certains acides aminés (tyrosine, phénylalanine) avec le niveau d'apports énergétiques bas, ce qui peut traduire un accroissement de leur całabolisme, mais aussi une réduction de leur apport dans l'intestin.

\section{Conclusions.}

Les effets possibles des niveaux d'apports énergétiques distribués au milieu et à la fin de la gestation sur le poids à la naissance et éventuellement sur la croissance ultérieure des agneaux sont bien démontrés ; ils existent même lorsque les écarts entre ces niveaux restent faibles, et lorsque les apports les plus bas sont encore compatibles avec le maintien de l'état de santé des brebis et des agneaux.

Les divergences observées, notamment suivant la saison d'agnelage nécessitent encore d'autres études, car les 2 expériences dont nous rapportons les résultats n'avaient pas été conduites dans ce but. Il en est de même pour les effets relatifs aux apports azotés et à leurs interactions avec les apports énergétiques.

4e Réunion du groupe Développement I.N.R.A. Montpellier, 17-18 mai 1978.

\section{Références}

CURLL M. L., DAVIDSON J. L., FREER M., 1975. Efficiency of lambs production in relation to the weight of ewe at mating and during pregnancy. Aust. J. agric. Sci., 26, 553-565.

EVERITT O. C., 1964. Maternal undernutrition and retarded foetal development in Merino sheep. Nature (Lond.). $201,1341$. 
EVERITT O. C., 1967. Residual effects of prenatal nutrition on the post natal performance of Merino sheep. Proc. N. Z. Soc. Anim. Prod., 27, 52.

GRIZARD J., TISSIER M., CHAMPREDON C., PRUGNAUD J., PION R., 1979. Variations des teneurs sanguines en acides aminés libres, urée et glucose chez la brebis en fin de gestation et début de lactation. Influence de l'état nutritionnel en fin de gestation. Ann. Biol. anim. Bioch. Biophys., 19, 55-71.

JARRIGE R., JOURNET M., VERITE R., 1978. Azote, 89-128. In Alimentation des Ruminants. Ed. INRA Publications (Route de Saint-Cyr), 78000 Versailles.

LOUCA A., MAVROGENIS A., LAWLOR M. J., 1974. Effects of plane of nutrition in late pregnancy on lamb birth-weight and milk yield in early lactation of Chios and Awassi sheep. Anim. Prod., 19, 341-349.

PEART J. N., 1967. The effect of different levels of nutrition during late pregnancy on the subsequent milk production of Blackface ewes and the growth of their lambs. J. agric. Sci., Camb., 68, 365-371.

PEART J. N., 1970. The influence of live-weight and body condition on the subsequent milk production of Blackface ewes following a period undernourishment in early lactation. J. agric. Sci., Camb., 75, 459-469.

PURROY U. A., 1978. Mesure de la composition corporelle des brebis à différents stades du cycle de reproduction par la méthode des espaces de diffusion. Thèse Doct. $3^{\mathrm{e}}$ cycle. Univ. Clermont.

TREACHER T. T., 1970. Effect of nutrition in late pregnancy on subsequent milk production in ewes. Anim. Prod., 12, 23-36.

WALLACE L. R., 1948. The growth of lambs before and after birth in relation to the level of nutrition. J. agric. Sci., Camb., 38, 93-398. 